\title{
Sur la rétention des particules de grande taille dans le réticulum chez la vache
}

C. DARDILLAT, R. BAUMONT

Station de Recherches sur la Nutrition des Herbivores I.N.R.A. Theix, 63122 Ceyrat, France.

Summary. To explain the retention of large particles in the reticulum, in vitro sedimentation, sorting of particles by gaz production and flow of contents through hole simulating the reticulo-omasal orifice (ROO) were examined. Removal of large particles from the floor of the reticulum by gaz production and a sieving mechanism through the ROO are discussed.

L'orifice reticulo-omasal (ORO) ne s'ouvre qu'au moment du transfert du contenu réticulaire (CR) dans l'atrium (Mc Bride et al., 1984) et seules les particules de petite taille passeraient dans l'omasum par un mécanisme de tri encore mal élucidé (Moir, 1984). Pour expliquer ce phénomène nous avons étudié les caractéristiques hydrodynamiques suivantes du CR : sédimentation ou flottaison des particules, séparation éventuelle des phases liquide et solide au cours de l'écoulement à travers un orifice.

Matériel et méthodes. Tout le CR $(5 I \pm 1)$ est prélevé par aspiration juste avant ou trois heures après la distribution de foin de graminées ou de luzerne chez une vache de $700 \mathrm{~kg}$ munie d'une canule du rumen. Deux litres de $\mathrm{CR}$ à $39^{\circ} \mathrm{C}$ placé pendant 10 min dans une éprouvette munie d'orifices latéraux sont séparés en fractions supérieures moyenne et inférieure, puis tamisés par voie humide à travers des grilles de $4,2,1,0,40,0,25,0,12$ et $0,05 \mathrm{~mm}$. Dans l'éprouvette les particules se déplacent sous les effets de leur densité relative, de l'entraînement par les bulles de gaz dégagées, et en fonction de leur résistance de frottement avec le liquide et surtout entre elles. Pour analyser l'importance respective de ces effets, nous avons répété l'expérience avec du contenu dégazé par le vide et refroidi à $10^{\circ} \mathrm{C}$ pour arrêter la fermentation. La simulation de l'aspiration du CR à travers I'ORO est obtenue par le montage suivant: du CR à $39^{\circ} \mathrm{C}$ placé dans une cuvette de $40 \mathrm{~cm}$ est mis en rotation ( 1 tour/s) par un moulinet axial de $38 \mathrm{~cm}$ de diamètre et s'écoule par un orifice latéral de $2 \mathrm{~cm}$.

Résultats et discussion. Dans l'éprouvette à $39^{\circ} \mathrm{C}$ les grandes particules (> à $4 \mathrm{~mm}$ ) s'accumulent dans la fraction supérieure (tabl. 1). L'appauvrissement en grandes particules de la fraction inférieure, voire moyenne, est plus rapide après le repas, pour la luzerne et pour une faible teneur en matière sèche. Sur du CR dégazé, la densité des particules $(1,20 \pm 0,05)$ exclut toute flottaison, mais la vitesse de sédimentation est trop faible pour être mesurable en $10 \mathrm{~min}$. Par contre sur des particules isolées, cette vitesse, fonction croissante de leur taille 
varie de 0,1 à $5 \mathrm{~cm} / \mathrm{s}$. L'accumulation des grandes particules dans la fraction supérieure est donc la conséquence de l'entraînement préférentiel de celles-ci par les gaz de fermentations en partie issus de la dégradation des bicarbonates salivaires. Dans le contenu sous-jacent plus fluide, une sédimentation peut alors s'effectuer.

TABL. 1. - Répartition (\% de la matière sèche) des particules selon leur taille dans les fractions supérieure (S), movenne $(\mathrm{M})$ et inférieure $(\mathrm{I})$ après $10 \mathrm{~min}$ de sédimentation à $39^{\circ} \mathrm{C}$ ou dans la fraction écoulée (E) à travers un orifice simulant l'ORO (chaque valeur est la moyenne de 3 répétitions).

\begin{tabular}{|c|c|c|c|c|c|c|c|c|c|c|c|c|c|c|c|c|c|}
\hline \multirow{3}{*}{ Particules } & \multicolumn{7}{|c|}{ Foin de graminées } & \multicolumn{10}{|c|}{ Foin de luzerne } \\
\hline & \multicolumn{4}{|c|}{ A jeun } & \multicolumn{3}{|c|}{ Nourri } & & \multirow[b]{2}{*}{$\mathrm{s}$} & \multicolumn{3}{|c|}{ A jeun } & \multicolumn{4}{|c|}{ Nourri } & \\
\hline & $S$ & $M$ & 1 & $E$ & $S$ & $M$ & 1 & & & $M$ & 1 & $E$ & $S$ & $M$ & 1 & E & \\
\hline & & 6,2 & 5,1 & 3,2 & 9,4 & 0,4 & 2,8 & & 7,0 & & 3 & & & 3,1 & & 2 & 3 \\
\hline & 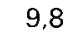 & 8,91 & 11,9 & 8,0 & 8,5 & & & & & & & & & & & & \\
\hline 0,0 & 35,03 & 34,43 & 32,93 & 32,33 & 30,12 & 27,82 & 29,32 & 27,23 & 31 & 30 & 34 & 30 & 51 & 16,52 & 27 & 24,9 & 6,7 \\
\hline Teneur en MS (\%) & 5,9 & 5,5 & 5,3 & 5,3 & 5,7 & 5,0 & 5,0 & 4,6 & 5,3 & 5,4 & 5,5 & 5,4 & 4,3 & 3,4 & 4,5 & 4,3 & 1,3 \\
\hline
\end{tabular}

PPDS : plus petite différence significative calculée avec $42 \mathrm{ddl}$. ( $n=48$ ).

L'écoulement par l'orifice du montage simulant le transit à travers l'ORO s'effectue par un jet de 20 à $200 \mathrm{ml}$ pendant 1 à $3 \mathrm{~s}$, brutalement interrompu par l'agrégation des particules sur l'orifice. Sa fermeture permet à l'agrégat d'être entraîné par le courant et à son ouverture le même phénomène se reproduit. In vivo, la fermeture de l'ORO (Mc Bride et al., 1984) accompagnée d'un reflux (Deswysen, 1985 ; Dardillat, 1987) agit probablement de façon similaire pour éviter son colmatage. Le contenu qui s'écoule est remarquablement appauvri en grandes particules (tabl. 1). Cet effet traduit la différence de vitesse d'écoulement entre la phase liquide (riche en petites particules) et la phase solide qui est freinée par l'adhérence aux parois du récipient. II s'observe pour l'écoulement d'une suspension dans un tuyau transparent et disparaît lorsque la viscosité de la phase liquide est artificiellement augmentée.

En conc/usion, la rétention des grandes particules dans le reticulum a pour origine leur flottaison sous l'effet du dégagement gazeux, à laquelle s'ajoute la filtration par l'ORO. La teneur en matière sèche et la viscosité de la phase liquide modifient l'influence de ces facteurs de rétention.

Dardillat C., 1987. Reprod. Nutr. Dévelop., 27, $231-232$.

Deswysen A. G., 1985, pp. 137-158. In The ruminant stomach. L.A.A. Ooms, A. D. Degryse, R. Marshboom (Ed.) Proc. Int. Workshop, Antwerp.

Mc Bride B. N., Milligan L. P., Turner B. V., 1984. Can. J. anim. Sci., 64, (Suppl.), 84-85.

Moir R. J., 1984 . pp. 85-92. In Ruminant physiology, concepts and consequences. S. K. Baker, J. M. Gathorne, J. B. Mackintosh, D. B. Purser (Ed.) Proc. Symp. Univ. Western Australia. Sydney. 